\title{
Hand Pose Estimation by Fusion of Inertial and Magnetic Sensing Aided by a Permanent Magnet
}

Henk G. Kortier, Jacob Antonsson, H. Martin Schepers, Fredrik Gustafsson and Peter H. Veltink

\section{Linköping University Post Print}

\section{Tweet}

N.B.: When citing this work, cite the original article.

Henk G. Kortier, Jacob Antonsson, H. Martin Schepers, Fredrik Gustafsson and Peter H. Veltink, Hand Pose Estimation by Fusion of Inertial and Magnetic Sensing Aided by a Permanent Magnet, 2015, IEEE transactions on neural systems and rehabilitation engineering, (23), 5, 796-806.

\section{http://dx.doi.org/10.1109/TNSRE.2014.2357579}

C2015 IEEE. Personal use of this material is permitted. However, permission to reprint/republish this material for advertising or promotional purposes or for creating new collective works for resale or redistribution to servers or lists, or to reuse any copyrighted component of this work in other works must be obtained from the IEEE.

\section{http://ieeexplore.ieee.org/}

Postprint available at: Linköping University Electronic Press

http://urn.kb.se/resolve?urn=urn:nbn:se:liu:diva-121903 


\title{
Hand pose estimation by fusion of inertial and magnetic sensing aided by a permanent magnet
}

\author{
Henk G. Kortier Student Member, IEEE, Jacob Antonsson, H. Martin Schepers, \\ Fredrik Gustafsson Fellow, IEEE, and Peter H. Veltink Senior Member, IEEE
}

\begin{abstract}
Tracking human body motions using inertial sensors has become a well-accepted method in ambulatory applications since the subject is not confined to a lab-bounded volume. However, a major drawback is the inability to estimate relative body positions over time because inertial sensor information only allows position tracking through strapdown integration, but doesn't provide any information about relative positions. In addition, strapdown integration inherently results in drift of the estimated position over time. We propose a novel method in which a permanent magnet combined with 3D magnetometers and 3D inertial sensors are used to estimate the global trunk orientation and relative pose of the hand with respect to the trunk. An Extended Kalman Filter is presented to fuse estimates obtained from inertial sensors with magnetic updates such that the position and orientation between the human hand and trunk as well as the global trunk orientation can be estimated robustly. This has been demonstrated in multiple experiments in which various hand tasks were performed. The most complex task in which simultaneous movements of both trunk and hand were performed resulted in an average rms position difference with an optical reference system of $19.7 \pm 2.2 \mathrm{~mm}$ whereas the relative trunk-hand and global trunk orientation error was $2.3 \pm 0.9$ and $8.6 \pm 8.7 \mathrm{deg}$ respectively.
\end{abstract}

Index Terms - sensor fusion, human body motion tracking, inertial sensing, magnetic tracking, upper extremity tracking

\section{INTRODUCTION}

Human body motion tracking is of wide interest in various areas, like sports, rehabilitation, ergonomics and entertainment industry [1] [2]. Traditionally, optical tracking systems are used to capture human body motions. However, they suffer from line of sight issues, non-portability and therefore operating such devices is often constrained to the volume in which they have been calibrated.

In the last decade, micro electrical mechanical system (MEMS) based inertial sensors became increasingly popular to employ on the human body and formed an alternative for motion tracking purposes [3] [4]. Besides the advantages compared to optical systems, inertial sensors introduce large estimation errors, for both orientation and position, due to integration of inertial signals.

H.G. Kortier and P.H. Veltink are with MIRA institute of Biomedical Technology and Technical Medicine, University of Twente, $7500 \mathrm{AE}$ Enschede, The Netherlands h.g.kortierdutwente.nl, p.h.veltink@utwente.nl

J. Antonsson is with the automatic control group, Lund University, SE22100 Lund, Sweden jacobadcontrol. lth. se

H.M. Schepers is with Xsens Technologies B.V., 7521 PR Enschede, The Netherlands hmartinschepers@gmail. com

F. Gustafsson is with the automatic control group, Linköping University, SE-581 83 Linköping, Sweden fredrik@isy. liu.se
For the estimation of drift free body orientations, several research groups proposed an Inertial and Magnetic Measurement System (IMMS) which is a filter framework to fuse inertial and magnetic information [5] [6] [7].

However, contrary to estimating 3D orientations, drift free estimates of 3D position over long measurements intervals is much more challenging, although important in many applications especially if non rigid segments as trunk and shoulder are involved.

An example is the assessment of reaching and grasping tasks which frequently performed in rehabilitation programs to address the severity of a certain disease. Outcome measures include the position accuracy, duration and smoothness of the hand's trajectory which is often addressed manually by a physician. Those parameters could be determined using an onbody sensing system which eventually result in a quantitative assessment. This can be obtained in the rehab centre or even at home in daily life situations, for instance by stroke patients [8].

For short time intervals in which the velocity of a certain limb can repeatedly be considered as negligible, for example the foot during walking, suitable initial and final conditions can be applied to mitigate integration drift of the estimated position [9] [10] [11].

Applying forward kinematics for articulated bodies seems to be a suitable method for estimation of positions when the orientation of each segment can be estimated and segmental lengths are known [12] [13]. However, the position error of the end effector accumulates along the articulated chain due to uncertainties in measured segmental lengths, sensor to segment calibration and joint models.

The only robust solution is to fuse inertial sensors with a position aiding system such as optical [14], acoustical [15], gps [16], uwb [17] or visual [18]. A magnetic tracking aiding system offers advantages compared to the other approaches, as indoor environments do not cause a degraded signal and there are no line of sight issues since the human body is transparent for magnetic fields [19].

However, magnetic fields easily get distorted in the vicinity of ferro-magnetic materials. Again, a possible solution is fusion with an aiding system, which eventually mitigates deviations from the correct state vector during magnetic field disturbances. A solution using an optical system was proposed by Vacarella et al. [25] and a solution using an inertial aiding system was proposed by Roetenberg et al. [5].

Latter method used actuated coils with inertial sensors embodied in a loose fusion filter using a magnetic dipole model. Schepers et al. [20] [21] proposed a similar method but 
improved the system such that many drawbacks, like coil constellation, energy consumption, loss of stochastic information and short distance measurements were tackled. Both methods resulted in an accurate position tracking system that can be used in an ambulatory setting.

However, some drawbacks still exists. First, energy consumption can be large, especially when large distances should be covered, and therefore limit the measurement time when the system is used in an ambulatory setting. Secondly, the coils can be rather large and heavy, which might result in an impaired movement or it could hinder the attachment of the source to specific body locations.

Finally, it was impossible to track rapid movements due to the inability of actuating the coils at a high rate and in parallel.

The idea of using a permanent magnet for localization and tracking was proposed by Birsan [22]. Using a permanent magnet as a source is beneficial compared to an active source as it can be kept small, works passively and is therefore more suitable for attachment on various body parts. A dipole model of a ferromagnetic object for the detection and tracking of metallic targets, particularly cars, is described in [23]. Based on this model Gustafsson and Wahlstrom developed a method to track $3 \mathrm{D}$ positions and $2 \mathrm{D}$ orientations using a grid of magnetometers and a permanent magnet acting as a source [24].

In this study we propose a new method which combines tracking of a permanent magnet using 3D magnetometers with inertial sensing. Hence, magnetic tracking results in drift free position estimates over long intervals ( $>1$ minute) which is impossible by solely using commercial grade inertial sensors, whereas inertial sensing allows for a robust $3 \mathrm{DoF}$ global and relative orientation estimate. In addition, inertial sensing gives pose information during short periods in which magnetometer information is lacking, for instance when the magnet is out of reach.

The aim of this study is to track 3D orientation and 3D position of the hand with respect to the trunk as well as the global orientation of the trunk. This is done by attaching a permanent magnet and an inertial sensor to the hand, and attaching multiple 3D magnetometers and an inertial sensor to the sternum.

\section{Methods}

Fig. 1 shows the measurement setup which consists of two parts, first the base $\left(\Psi_{b}\right)$, comprises a constellation of one or more $(l)$ rigidly connected 3D magnetometers. In addition, inertial information of this constellation is obtained using a $3 \mathrm{D}$ gyroscope and 3D accelerometer.

Secondly, the target $\left(\Psi_{t}\right)$, contains a rigidly connected 3D accelerometer, 3D gyroscope and a permanent magnet. It is the objective to track the position $\boldsymbol{p}_{t}^{b}$ and orientation $q^{b t}$ of the target with respect to the base and the orientation of the base with respect to a global frame $q^{g b}$. A general schematic layout is depicted in Fig. 2. One can see that it is necessary to have prior knowledge about the constellation. More specifically, the relative position and orientation of all magnetometers with respect to the base frame, as well as the

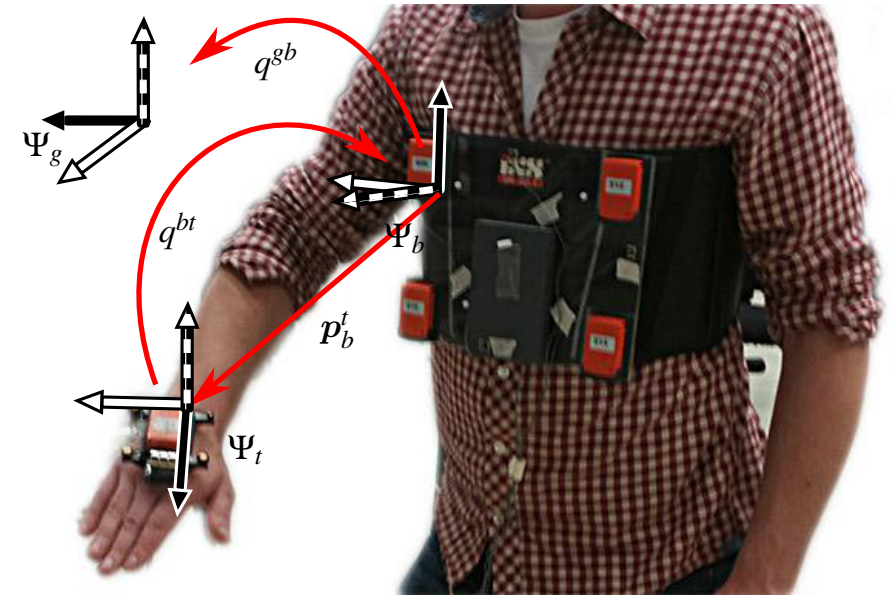

Fig. 1: Tracking instrumentation attached to trunk and right hand. Visible are four IMMS's (orange) attached to the trunk, each containing a 3D magnetometer. The IMMS that is positioned closest to the right shoulder acts as the primary one and is therefore designated as the trunk's reference frame $\left(\Psi_{b}\right)$. Furthermore, accelerometer and gyroscope data is acquired from this IMMS. On the hand, a single IMMS together with a neodymium magnet (silver grey) is visible. The position of the hand $\left(\boldsymbol{p}_{b}^{t}\right)$ and orientation $\left(q^{b t}\right)$ with respect to the trunk is being estimated. Furthermore the orientation of the trunk with respect to a global frame $\left(\Psi_{g}\right)$ is estimated. For each coordinate frame the $\mathrm{X}$ (black), Y (white) and $\mathrm{Z}$ (dashed) directions are indicated.

position and orientation of the magnet with respect to the local inertial sensor should be known.

The following sections will describe the filter structure, the measurement models, the process model and finally the experimental methods which are used to validate the accuracy of the proposed system.

\section{A. Filter structure}

An Extended Kalman Filter (EKF) has been deployed in order to estimate relative positions as well as relative and absolute orientations [26] [27], see Fig. 3. Inertial measurements are primarily used as an input for the process dynamics whereas the magnetic measurement are used for correction. The state space equations are given by:

$$
\begin{aligned}
\boldsymbol{x}_{k+1} & =f\left(\boldsymbol{x}_{k}, \boldsymbol{u}_{k}\right)+\boldsymbol{w}_{k} \\
\boldsymbol{y}_{k} & =h\left(\boldsymbol{x}_{k}\right)+\boldsymbol{v}_{k}
\end{aligned}
$$

where $f\left(\boldsymbol{x}_{k}, \boldsymbol{u}_{k}\right)$ denotes the process model, $\boldsymbol{y}_{k}$ the measurements, and $h\left(\boldsymbol{x}_{k}\right)$ the measurement model. Process and measurement noise are assumed to be independent identically distributed (i.i.d.) Gaussian noise, denoted by $\boldsymbol{w}_{k}$ and $\boldsymbol{v}_{k}$.

The measurement vectors at time $k$ includes $l$ magnetometers $\boldsymbol{y}_{m, k}^{\left\{b_{1} . b_{l}\right\}}$ and two accelerometers $\boldsymbol{y}_{a, k}^{\{b, t\}}$. In addition, the accelerometers are used together with two gyroscopes $\boldsymbol{y}_{\omega, k}^{\{b, t\}}$ as an input $\boldsymbol{u}_{k}$. 


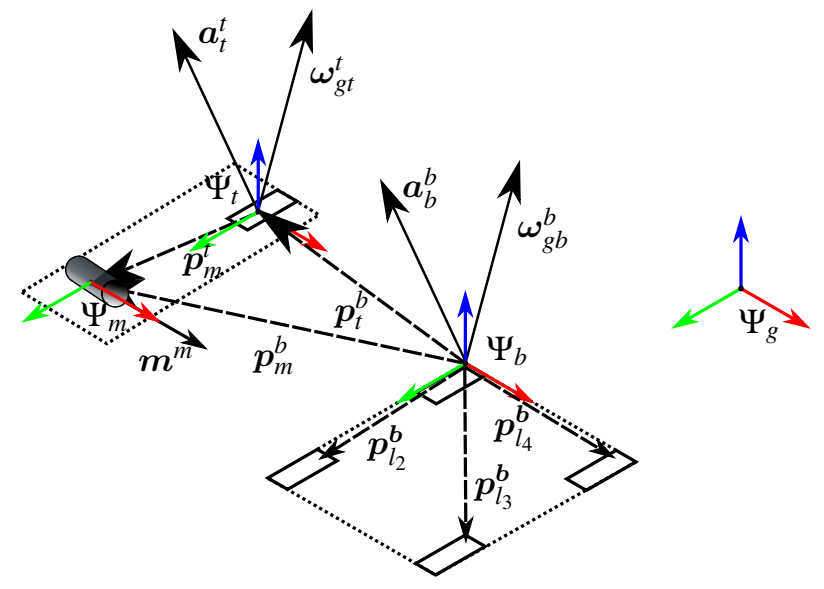

Fig. 2: Schematic diagram illustrating the global frame $\Psi_{g}$, base frame $\Psi_{b}$ and the rigidly attached target frame $\Psi_{t}$ and permanent magnet frame $\Psi_{m}$. The relative position $\boldsymbol{p}_{t}^{b}$ and orientation $q^{b t}$ as well as the global orientation $q^{b g}$ are estimated by the filter. The base is constellated by $l 3 \mathrm{D}$ magnetometers of which their position and orientation with respect to the first magnetometer is invariant and known priorly. In addition, the local magnetic moment $\left(\boldsymbol{m}^{m}\right)$, inertial acceleration and angular velocity of target $\left(\boldsymbol{a}_{t}^{t}, \boldsymbol{\omega}_{g t}^{t}\right)$ and base $\left(\boldsymbol{a}_{b}^{b}, \boldsymbol{\omega}_{g b}^{b}\right)$ are given.

The state vector includes the following elements:

$$
\boldsymbol{x}=\left[\begin{array}{llllllll}
\boldsymbol{p}_{t}^{b} & \boldsymbol{v}_{t}^{b} & \delta \boldsymbol{\theta}^{b t} & \boldsymbol{B}^{g} & \boldsymbol{b}_{g}^{t} & \boldsymbol{b}_{a}^{t} & \delta \boldsymbol{\theta}^{g b} & \boldsymbol{b}_{g}^{b}
\end{array}\right]^{T}
$$

where $\boldsymbol{p}_{t}^{b}$ and $\boldsymbol{v}_{t}^{b}$ are the position and velocity of the target expressed in the base frame respectively, $\boldsymbol{B}^{g}$ is the environmental magnetic field experienced by the base expressed in global frame, $\boldsymbol{b}_{g}^{t}$ and $\boldsymbol{b}_{a}^{t}$ are the gyroscope and accelerometer bias of the target sensor respectively and $\boldsymbol{b}_{g}^{b}$ is the gyroscope bias attached to the base.

Both orientations are expressed as a unit quaternion $\left(q^{b t}\right.$, $\left.q^{g b}\right)$, and therefore require the unity norm constraint. Because an EKF is not suitable to handle such constraints properly, the true quaternion is parameterised using a nominal $\bar{q}$ and error part $\delta q$ [27]. Under the assumption that the error part is small, we can approximate the error quaternion using an error angle representation $\delta \boldsymbol{\theta}$ :

$$
\begin{aligned}
q & =\bar{q} \odot \delta q \\
& \approx \bar{q} \odot\left[1 \quad \frac{1}{2} \delta \theta\right]^{T}
\end{aligned}
$$

where $\odot$ is the quaternion product operator [28]. The error angles can be handled properly by the EKF filter and therefore included in the state vector $\left(\delta \boldsymbol{\theta}^{b t}, \delta \boldsymbol{\theta}^{g b}\right)$. The corresponding quaternions are adjusted (Fig. 3. ORIENT UPDATE) after each measurement update step using the error angle estimates. Because propagation of the error angle (see section II-C) is a function of the gyroscope's error bias, a similar approach for the gyro biases is taken:

$$
\boldsymbol{b}_{g}=\overline{\boldsymbol{b}}_{g}+\delta \boldsymbol{b}_{g}
$$

where $\boldsymbol{b}_{g}$ is the true gyro bias which is modeled by a nominal $\left(\overline{\boldsymbol{b}}_{g}\right)$ and error part $\left(\delta \boldsymbol{b}_{g}\right)$. The latter is included in the state

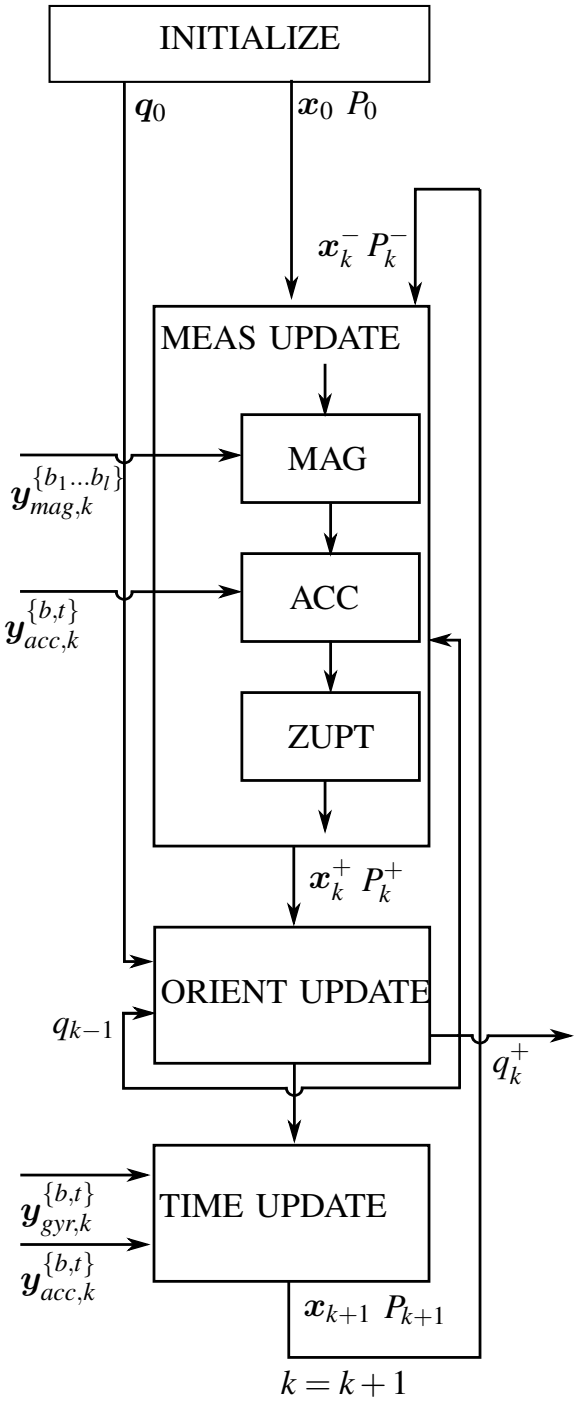

Fig. 3: Topology of the implemented EKF. After initialisation of both state $\boldsymbol{x}_{0}$ and orientations $q_{0}$ and corresponding covariance $P_{0}$, a measurement update is performed. This step includes a magnetic update (MAG), acceleration update (ACC) and, when applicable, a zero velocity update (ZUPT). The magnetic update uses information obtained from $l$ magnetometers expressed in base frame $\Psi_{b}$. The acceleration update step uses accelerometer information of both base and target. Finally the zero velocity update applies an update when either the target velocity is zero with respect to base or when the target exceeds a pre-defined measurement volume. After the measurement update, the orientation estimates $q$ of both trunk and target are updated using the estimated error angles $\delta \boldsymbol{\theta}$. Subsequently, a time update is performed which includes propagation of the state with corresponding covariance.

vector and used to update the true bias after the measurement updates. Finally, the error angle and gyro error bias estimates are set to zero before propagated by the process model.

The measurement model is divided in a magnetic update $h_{\text {mag }}$, acceleration update $h_{a c c}$ and zero velocity update $h_{z u p t}$. The magnetic update provides information of the relative hand position, $2 \mathrm{DoF}$ of the relative orientation and heading 
information of the trunk, see section [II-B1] The acceleration update is required to ensure observability of target and trunk inclination, see section II-B2 Finally, the zero velocity update is used to provide additional information in specific conditions, which will be explained in section II-B3

The a-posteriori state $\left(\boldsymbol{x}_{k}^{+}\right)$and corresponding covariance estimate $\left(P_{k}^{+}\right)$, given the a-priori state and covariance, is determined using the measurement function $\left(h\left(\boldsymbol{x}_{k}^{-}\right)\right)$its corresponding linearization $\left(H_{k}\right)$ and calculated Kalman gain $\left(K_{k}\right)$ [26]:

$$
\begin{aligned}
\boldsymbol{x}_{k}^{+} & =\boldsymbol{x}_{k}^{-}+K_{k}\left(\boldsymbol{y}_{k}-h\left(\boldsymbol{x}_{k}^{-}\right)\right) \\
P_{k}^{+} & =\left(I-K_{k} H_{k}\right) P_{k}^{-} .
\end{aligned}
$$

Propagation of position and orientation change is obtained by integration of the relative angular velocity and double integration of the relative acceleration, which is described in section II-C The uncertainty of the corresponding state is propagated according to:

$$
P_{k+1}^{-}=F_{k} P_{k}^{+} F_{k}^{T}+Q_{k}
$$

where $F_{k}$ the linearized process model $f\left(\boldsymbol{x}_{k}, \boldsymbol{u}_{k}\right)$ and $Q_{k}$ the covariance matrix of the process noise. The initial covariance $P_{0}$ is chosen large for all states except for the relative velocity and orientations because the movement is initiated from rest an arbitrary location. The process noise covariance $Q_{k}$ is experimentally determined by estimating the standard deviation of the inertial sensors.

\section{B. Measurement models}

1) Magnetic model: A dipole model is used to track $2 \mathrm{DoF}$ orientation and 3 DoF position of the target [23] [29], and heading information of the trunk. The output of each magnetometer attached to the base is modeled as:

$$
\begin{aligned}
\boldsymbol{y}_{m}^{l} & =h_{m}^{l}(\boldsymbol{x})+\boldsymbol{e}_{m} \\
& =\boldsymbol{B}+\boldsymbol{J}\left(\boldsymbol{r}^{l}\right) \boldsymbol{m}^{l}+\boldsymbol{e}_{m}
\end{aligned}
$$

where the superscript $l$ indicates the particular magnetometer and $\boldsymbol{r}^{l}$ its position with respect to the permanent magnet. The measured field exists of a common component $\boldsymbol{B}$ and a position dependent component $J(\boldsymbol{r})$ which is given by a magnet dipole model:

$$
J(\boldsymbol{r})=\frac{1}{\|\boldsymbol{r}\|_{2}^{5}}\left(3 \boldsymbol{r} \boldsymbol{r}^{T}+\|\boldsymbol{r}\|_{2}^{2} I_{3}\right) .
$$

The position of the target expressed in the base frame is given by (see Fig. 2):

$$
\boldsymbol{p}_{t}^{b}=\boldsymbol{p}_{m}^{b}-R^{b t} \boldsymbol{p}_{m}^{t}
$$

where $\boldsymbol{p}_{m}^{t}$ is the position of the magnet with respect to the local inertial sensor. Subsequently, the position of the magnet measured by magnetometer $l$ expressed in the frame $\Psi_{b}$ is given by:

$$
\begin{aligned}
\boldsymbol{r}^{b} & =\boldsymbol{p}_{m}^{b}-\boldsymbol{p}_{l}^{b} \\
& =\boldsymbol{p}_{t}^{b}+R^{b t} \boldsymbol{p}_{m}^{t}-\boldsymbol{p}_{l}^{b}
\end{aligned}
$$

where $\boldsymbol{p}_{l}^{b}$ is the position of magnetometer $l$ with respect to primary magnetometer expressed in base frame. The relative orientation between the permanent magnet and local target frame is given by $R^{t m}$. This magnetic moment vector $\boldsymbol{m}^{m}$ expressed in the base frame is given by:

$$
\boldsymbol{m}^{b}=R^{b t} R^{t m} \boldsymbol{m}^{m} .
$$

The global magnetic field $B^{g}$, which is assumed to be homogeneous within the measurement volume, expressed in the base frame is given by:

$$
\boldsymbol{B}^{b}=R^{b g} \boldsymbol{B}^{g}
$$

Substituting the parameterized orientation 3 into the equations (10), (11), (12) and using the assumption that the magnetometers are equally orientated $\left(R^{b l}=I_{3}\right)$ gives:

$$
\begin{aligned}
\boldsymbol{r}^{b} & =\boldsymbol{p}_{t}^{b}+\bar{R}^{b t}\left(I_{3}+[\boldsymbol{\delta} \boldsymbol{\theta}]_{\times}^{b t}\right) \boldsymbol{p}_{m}^{t}-\boldsymbol{p}_{l}^{b} \\
\boldsymbol{m}^{b} & =\bar{R}^{b t}\left(I_{3}+[\boldsymbol{\delta} \theta]_{\times}^{b t}\right) \boldsymbol{R}^{t m} \boldsymbol{m}^{m} \\
\boldsymbol{B}^{b} & =\bar{R}^{g b, T}\left(I_{3}-[\boldsymbol{\delta} \boldsymbol{\theta}]_{\times}^{g b}\right) \boldsymbol{B}^{g}
\end{aligned}
$$

where the time indices $k$ have been omitted for clarity and [] is used to denote a skew symmetric matrix.

In order to construct the Jacobian $H_{m a g}$, the partial derivatives with respect to the state vector are required. A detailed derivation can be found in appendix A

2) Acceleration model: The accelerometers on both base and target can be used to obtain a local inclination estimate. This is only valid in a static situation where the inertial acceleration is negligible. Large deviations, i.e. the norm of the measured accelerometer signal is not close to the gravitation constant and the angular velocity norm is not close to zero, can be detected using a Generalized Likelihood Ratio Test (GLRT). This concept has been described by Skog et.al. [9] and has been implemented such that for the "in movement" hypothesis the measurement covariance is adjusted accordingly. The threshold parameters of the GLRT are chosen such that accelerometer measurements are only included if their norm is within five percent of the gravity vector norm.

The measurement model is given by:

$$
\boldsymbol{h}_{a c c, b}^{\{b, t\}}=\boldsymbol{y}_{a c c}^{\{b, t\}}-\bar{R}^{\{b, t\} g}\left(I_{3}-[\delta \boldsymbol{\theta}]_{\times}^{g\{b, t\}}\right) \boldsymbol{g}^{g}+\boldsymbol{e}_{a}
$$

where $\boldsymbol{g}^{g}$ is the known gravitational acceleration vector expressed in the global frame, and $\boldsymbol{e}_{a}$ is the i.i.d. Gaussian noise.

However, if both base and target frame experience the same inertial acceleration, additional information about the relative heading can be provided. Therefore a different measurement model is used for the target:

$$
\boldsymbol{h}_{a c c, t}^{t}=\boldsymbol{y}_{a c c}^{t}-\bar{R}^{t b}\left(I_{3}-[\delta \theta]_{\times}^{b t}\right) \boldsymbol{y}_{a c c}^{b}+\boldsymbol{e}_{a} .
$$

The difference in magnitude of both measured accelerometer signals is used to test whether this update is applicable.

3) Zero velocity update: In order to mitigate position diverging, zero velocity updates are applied:

$$
\boldsymbol{h}_{z u p t}^{b}=\boldsymbol{v}^{b}+\boldsymbol{e}_{z}
$$

Two different conditions are tested. First, whenever the target or base does not experience an inertial acceleration it is assumed that either is held being still which is tested by the 
same GLRT used for the inclination update. Second, if the target position with respect to the trunk exceeds a predefined cubic volume, the estimate will be kept in this volume by setting the velocity to zero. Latter is used to make the filter more robust such that velocity and position do not drift to infinity when no magnetic information is available for long periods.

\section{Process model}

The velocity $\boldsymbol{v}_{t}^{b}$ and orientations $\left\{q^{g b}, q^{b t}\right\}$ are obtained by integration of the difference in measured acceleration $\boldsymbol{a}_{t}^{b}$ and angular velocity $\boldsymbol{\omega}_{b t}^{t}$. The difference in acceleration $\boldsymbol{a}_{t}^{b}$ can be expressed as a function of the measured accelerometer signals $\left(\boldsymbol{y}_{a c c}^{t}, \boldsymbol{y}_{a c c}^{b}\right)$ :

$$
\begin{aligned}
\boldsymbol{a}_{t}^{b} & =R^{b t} \boldsymbol{a}^{t}-\boldsymbol{a}^{b} \\
& =R^{b t}\left(\boldsymbol{y}_{a c c}^{t}-\boldsymbol{b}_{a, t}^{t}-R^{t g} \boldsymbol{g}^{g}\right)-\left(\boldsymbol{y}_{a c c}^{b}-\boldsymbol{b}_{a, b}^{b}-R^{b g} \boldsymbol{g}^{g}\right)+\boldsymbol{e}_{a} \\
& =R^{b t}\left(\boldsymbol{y}_{a c c}^{t}-\boldsymbol{b}_{a, t}^{t}\right)-\left(\boldsymbol{y}_{a c c}^{b}-\boldsymbol{b}_{a, b}^{b}\right)+\boldsymbol{e}_{a}
\end{aligned}
$$

where $\boldsymbol{b}_{a, t}^{t}$ is the accelerometer bias of the target and $\boldsymbol{b}_{a, b}^{b}$ the bias of the base accelerometer which is assumed to be negligible.

The difference in angular velocity expressed in the target frame is given by the difference in measured gyroscope signals of the target $\boldsymbol{y}_{g y r}^{t}$ and base $\boldsymbol{y}_{g y r}^{b}$ :

$$
\begin{aligned}
\boldsymbol{\omega}_{b t}^{t} & =\boldsymbol{\omega}_{g t}^{t}-R^{t b} \boldsymbol{\omega}_{g b}^{b} \\
& =\left(\boldsymbol{y}_{g y r}^{t}-\boldsymbol{b}_{g, t}^{t}\right)-R^{b t, T}\left(\boldsymbol{y}_{g y r}^{b}-\boldsymbol{b}_{g, b}^{b}\right)+\boldsymbol{e}_{g} .
\end{aligned}
$$

Propagation of the hand and trunk orientation is given by:

$$
\begin{aligned}
& q_{k+1}^{b t}=q_{k}^{b t} \odot\left(\frac{1}{2} T \boldsymbol{\omega}_{k, b t}^{t}\right) \\
& q_{k+1}^{g b}=q_{k}^{g b} \odot\left(\frac{1}{2} T \boldsymbol{\omega}_{k, g b}^{b}\right)
\end{aligned}
$$

where $k$ indicate the sample, $T$ is the sample period and $\odot$ is the quaternion product operator [28]. Subsequently, the linearized state propagation equations which eventually constitute the state transition matrix $F_{k}$ are stated as:

$$
\begin{aligned}
\boldsymbol{p}_{k+1}^{b} & =\boldsymbol{p}_{k}^{b}+\boldsymbol{T} \boldsymbol{v}_{k}^{b}+\frac{1}{2} T^{2} \boldsymbol{a}_{k, t}^{b} \\
\boldsymbol{v}_{k+1}^{b} & =\boldsymbol{v}_{k}^{b}+\boldsymbol{T} \boldsymbol{a}_{k, t}^{b} \\
\delta \boldsymbol{\theta}_{k+1}^{b t} & =\left(I_{3}-\left[T \boldsymbol{\omega}_{k, b t}^{b}\right]_{\times}\right) \delta \boldsymbol{\theta}_{k}^{b t}-T\left(\delta \boldsymbol{b}_{g}^{t}-\delta \boldsymbol{b}_{g}^{b}\right)+\boldsymbol{e}_{\theta} \\
\boldsymbol{B}_{k+1}^{g} & =\boldsymbol{B}_{k}^{g}+\boldsymbol{e}_{B} \\
\boldsymbol{b}_{a, k+1}^{t} & =\boldsymbol{b}_{a, k}^{t}+\boldsymbol{e}_{b a} \\
\delta \boldsymbol{b}_{g, k+1}^{t} & =\delta \boldsymbol{b}_{g, k}^{t}+\boldsymbol{e}_{b g} \\
\delta \boldsymbol{\theta}_{k+1}^{g b} & =\left(I_{3}-\left[T \boldsymbol{\omega}_{k, g b}^{b}\right]_{\times}\right) \boldsymbol{\delta} \boldsymbol{\theta}_{k}^{g b}-T \delta \boldsymbol{b}_{g}^{b}+\boldsymbol{e}_{\theta} \\
\delta \boldsymbol{b}_{g, k+1}^{b} & =\delta \boldsymbol{b}_{g, k}^{b}+\boldsymbol{e}_{b g} .
\end{aligned}
$$

A derivation of error angle propagation $\delta \boldsymbol{\theta}$ is given in the appendix C.

The local magnetic field $\boldsymbol{B}^{g}$, gyroscope error biases, $\delta \boldsymbol{b}_{g}^{t}$ $\delta \boldsymbol{b}_{g}^{b}$, and accelerometer bias $\left(\boldsymbol{b}_{a}^{t}\right)$ are modelled as random walk processes to account for their low pass behaviour.

\section{Experimental methods}

Four Anisotropic Magneto Resistive (AMR) magnetometers (Honeywell HMC5883L), each embodied in an IMMS (Xsens Technologies B.V. MTw), were rigidly attached to a PMMA panel and strapped to the subject's trunk, see Fig.11 One of the IMMS's was appointed as the primary sensor and designated as the origin of the trunks reference frame $\left(\Psi_{B}\right)$. In addition to the magnetometers, inertial sensor signals are obtained from the primary IMMS's accelerometer and gyroscope.

The target, which was placed on the hand, comprises a rigid plaster piece on which a magnet (neodymium rod, length: $2 \mathrm{~mm}$, radius: $7 \mathrm{~mm}$ SuperMagnete.de) and IMMS were attached. The position and orientation of the magnet with respect to the local accelerometer was estimated using a ruler beforehand.

All IMMS data was sampled at $60 \mathrm{~Hz}$ and transmitted wirelessly to the PC (Xsens Technologies B.V. Awinda). The sensors contain a rechargeable lithium-ion battery which will run for about 4 hours during measurements [30].

Prior to the experiments all magnetometers were calibrated within the volume used in the experiments using the magnetic field mapping procedure. All magnetometers were rigidly attached and rotated in any direction, subsequently the magnetic field vector are mapped onto a unit sphere using a maximum likelihood (ML) approach described by Kok et al. [31].

As the magnetometer output is normalized during this calibration procedure, we will define the SNR of each magnetometer as:

$$
S N R^{l}=20 \log \left(\left\|y_{\text {mag }}^{l}\right\|_{2}\right) .
$$

Also both trunk and target accelerometer were calibrated using a fairly simple least squares approach [32]. It should be noted that the bias of the base accelerometer has not been included in the state vector as it is not observable. Finally, the relative position and orientation between magnetometers attached to the plexi panel is required. Because the magnetometer housing also contains inertial sensors of which the position with respect to the local magnetometer is known, it is possible to solve for the relative position and orientation between the rigidly connected magnetometers by expressing the local acceleration as function of the orientation, angular velocity and acceleration and the relative position for each inertial sensor with respect to the other rigidly connected inertial sensors. Now, the required parameters can be obtained if the rigid body is sufficiently accelerated around each axis. A modified version of this algorithm described by Parsa et al. [33] was applied for this purpose.

Both rigid pieces on trunk and hand were accommodated with optical markers (PTI VisualEyez VZ-4000), such that position and orientations could be calculated and subsequently compared with our system.

The inertial sensor system and optical system were synchronized by maximising the correlation between the estimated angular velocities of the hand obtained from both systems.

Possible gaps of the optical system were spline-interpolated with a maximum size of 30 samples. 
During the measurement the subject performed various hand tasks while seated at a desk. The total experiment included the following measurements:

1) Static trunk, varying hand: The position and orientation of the trunk were (pseudo) static whereas the position and orientation of the hand were varying cyclically. The subject was asked to maximise the reaching area while seated and minimizing trunk rotations.

2) Varying trunk, static hand: The position and orientation of the trunk were varied by repeated rotations of the trunk around the longitudinal body axis. The subject was asked to keep the hand in a constant relative position and orientation with respect to the trunk.

3) Varying trunk and target. The subject was asked to mimic repeated pick and place actions which required him to change orientation of trunk and hand and relative position between trunk and hand simultaneously. There were no constraints with respect to either trunk or hand movement.

Each measurement lasted 70 seconds, was performed 5 times and all conducted by a single subject. The first $10 \mathrm{sec}-$ onds of each trial were not taken into account for comparison between our estimates and the optical reference, because this time was required for the filter to recover from an incorrect initial estimate.

\section{RESULTS}

A representative trial corresponding to the first measurement condition is depicted in Fig. 4 This figure represents the estimate of both distance and total orientation angles (axisangle representation, [28]) of the hand with respect to the trunk and the trunk with respect to the global frame. The axes of the trunk are defined such that $X$ points vertically upwards (cranial), $Z$ points in anterior direction (ventral) and $Y$ is chosen such that a right-handed coordinate frame is formed (see Fig. 11). The position error is defined as the difference between relative position as estimated by the proposed system and the optical reference system. Similarly, the orientation error is defined as the smallest angle about which the relative orientation of the sensor estimated by the ambulatory system has to be rotated to coincide with the relative orientation estimated by the reference system. The subject started with the hand far away from the magnetometers, and therefore a low SNR of the magnetic signal induced by the permanent magnet was obtained. In addition, the initial distance estimate was set to zero. For these reasons, the distance and relative orientation estimate up to approximately 5 seconds is unreliable. Periods in which the SNR was above $0.5 d B$ are indicated using a grey shaded background.

From Fig. 4 it is noticeable that in many periods minimal information from the permanent magnet was obtained because magnet was too far away from the trunk. This is certainly the case in the first experiment where the SNR was below $0.5 d B$ for $50.1 \pm 10.0 \%$ of the time compared to the second $(0.1 \pm 0.0 \%)$ and third experiment $(8.6 \pm 8.7 \%)$.

A representative trial of the third, and most complex, movement condition is presented in Fig. 5a and 6 The former
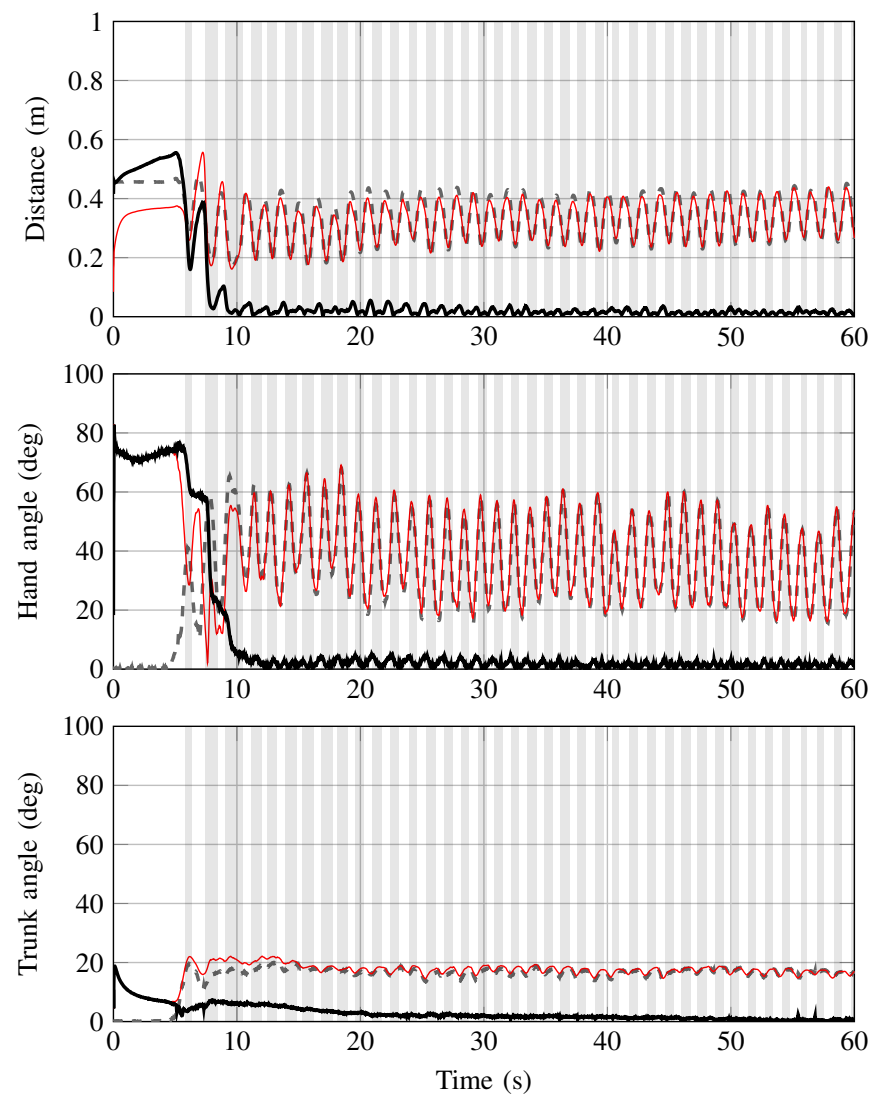

Fig. 4: Distance (top) and total angle (axis-angle representation) of the relative orientation (hand with respect to trunk) and global orientation (trunk with respect to the static environment) during the reaching task (condition 1). The plots show the estimated values (red), optical reference (grey dashed) as well the corresponding differences (black). An SNR above $0.5 d B$ induced by the permanent magnet is indicated (grey background).

figure shows the estimated position of the hand with respect to the trunk together with the optical reference whereas the latter figure shows both the orientation of the trunk with respect to the static global frame and the hand with respect to the trunk. The orientation is represented using Euler angles, in which pitch, roll and yaw represent an angle around the $X, Y$ and $Z$ axis, respectively.

In addition, an estimate of those three kinematic variables using the same measurement trial was made by using only one magnetometer (the one closest to the right shoulder, see Fig. 1 instead of four. The position is depicted in Fig. $5 \mathrm{~b}$ and the orientations in Fig. 7 Compared to the estimates using four magnetometer it is noticeable that the reconstruction of both position and orientations is worse when a single magnetometer is used, especially in low SNR periods. Furthermore, the angle around the vertical is hard to estimate (global yaw, trunk pitch), which can be explained by the fact that is impossible to distinguish between variations in environmental magnetic field and the induced magnetic field by the permanent magnet.

In Fig. 8 the error distributions of relative positions, orien- 
(a) Four magnetometers
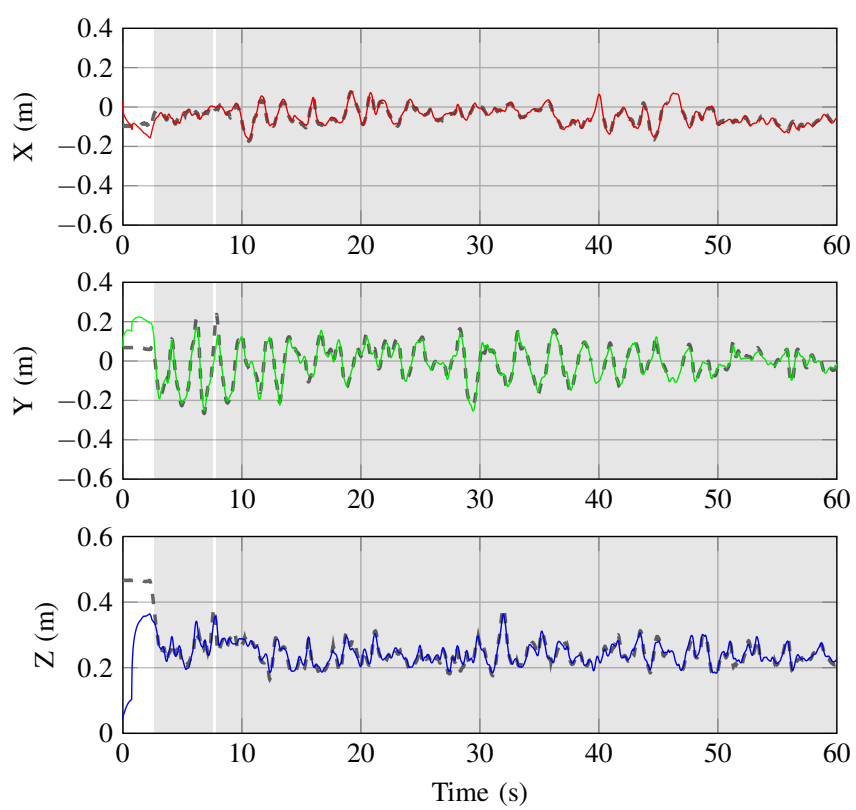

(b) One magnetometer
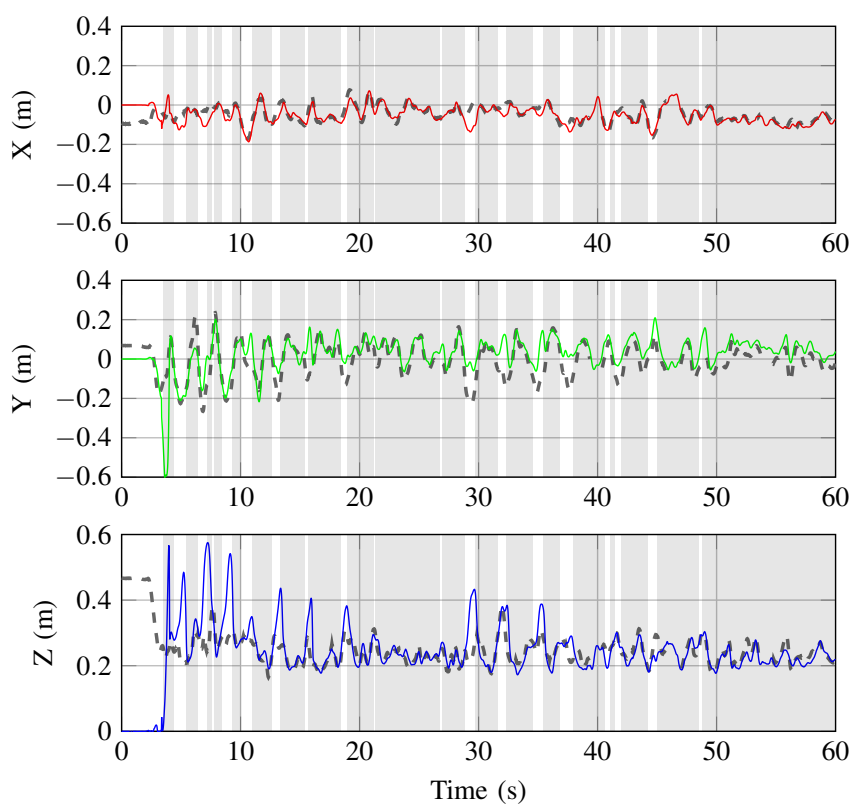

Fig. 5: Estimated position of a varying trunk and hand task (condition 3). Visible is the estimated position $(X, Y, Z)$ together with the optical reference (grey, dashed). Time periods in which the SNR induced by the permanent magnet exceeded $0.5 d B$ are indicated (grey background). Left (a): Four magnetometers used. Right (b): One magnetometer used.

(a) Global trunk orientation using four magnetometers
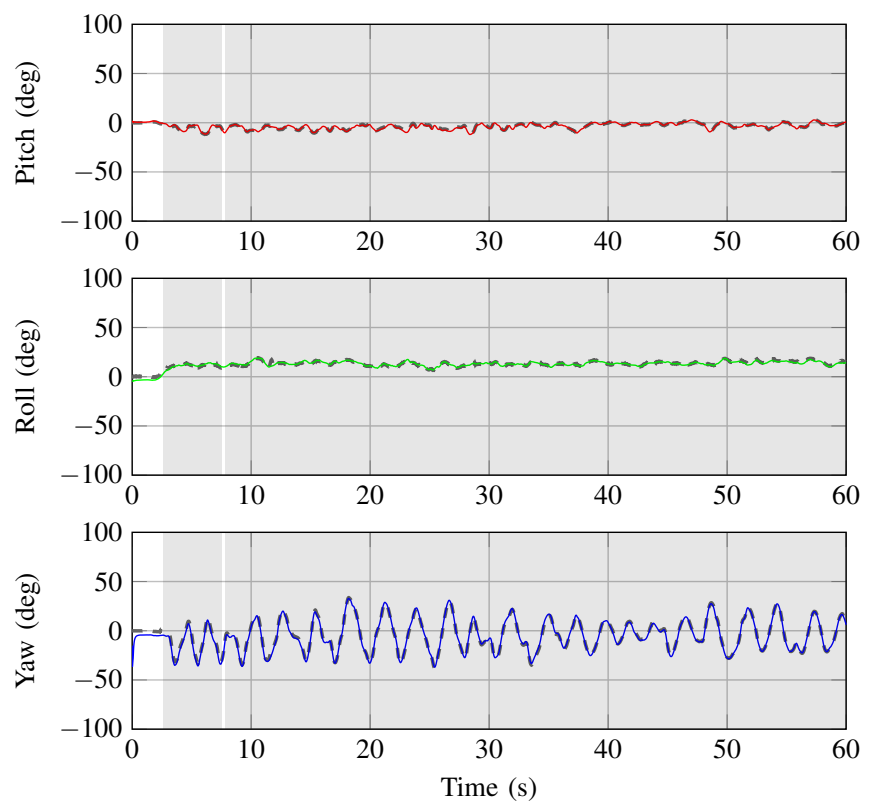

(b) Relative hand trunk orientation using four magnetometers
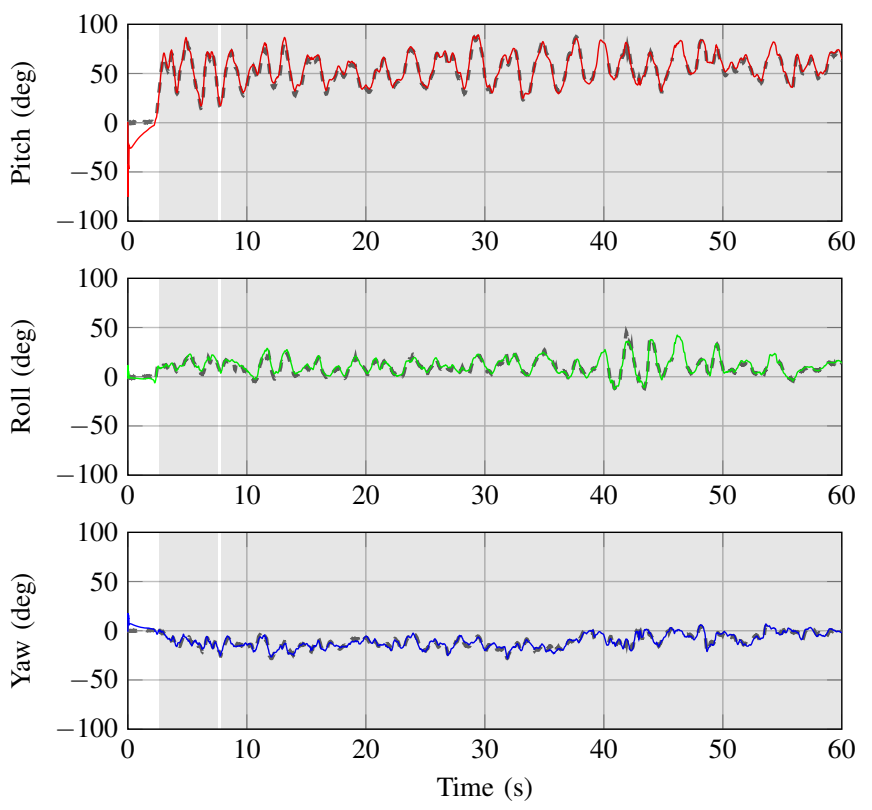

Fig. 6: Representative trial of a varying trunk and hand task (condition 3) with four magnetometers. Left (a): reconstruction of the absolute trunk orientation. Right $(b)$ : reconstruction of hand orientation with respect to the trunk. The orientation are expressed in euler angles (Pitch, Roll, Yaw) A comparison with an optical system is made (grey, dashed). Time periods in which the SNR induced by the permanent magnet exceeded $0.5 d B$ are indicated (grey background). 
(a) Global trunk orientation using one magnetometer
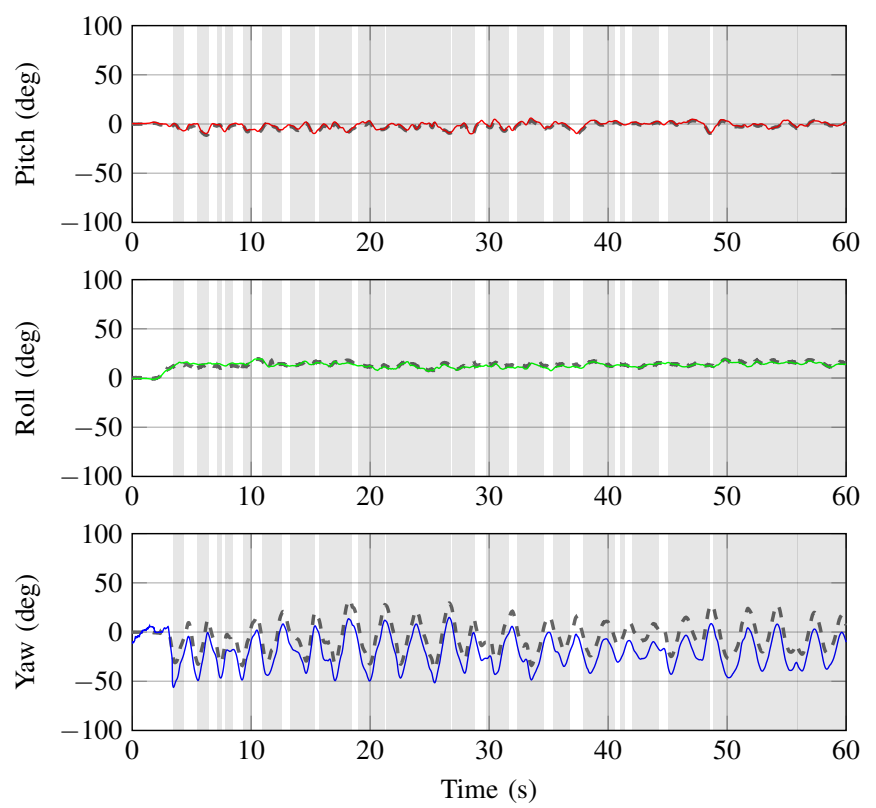

(b) Relative hand trunk orientation using one magnetometer
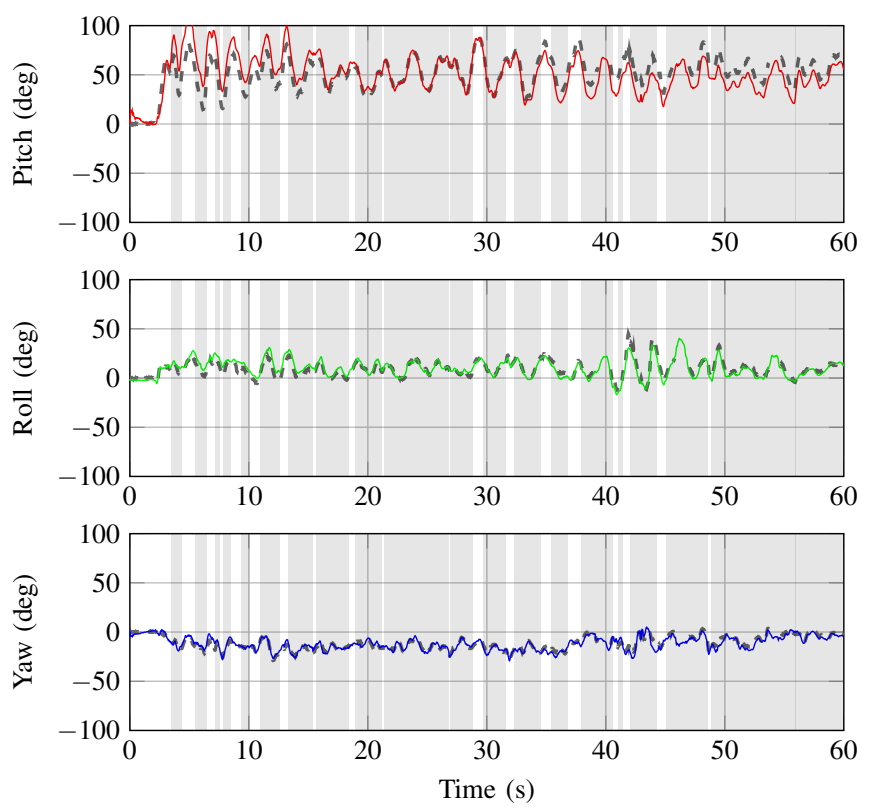

Fig. 7: Representative trial of a varying trunk and hand task (condition 3) with one magnetometer. Left (a): reconstruction of the absolute trunk orientation. Right $(b)$ : reconstruction of hand orientation with respect to the trunk. The orientation are expressed in euler angles (Pitch, Roll, Yaw) A comparison with an optical system is made (grey, dashed). Time periods in which the SNR induced by the permanent magnet exceeded $0.5 d B$ are indicated (grey background).

tations and absolute orientation are given for all measurements and represented using box whisker plots. The box plots illustrate that the errors in the position estimate for the second movement condition (varying trunk, static hand) are smaller compared to the other two movement conditions. However, the difference in relative orientation (trunk hand) is larger for this condition, which can be explained by a twice as large magnitude of the trunk's angular velocity compared to the other two conditions. The error in the global trunk orientation estimate is largest for the third measurement condition. This is presumably caused due the tight filter structure and complexity of movement. Simultaneous trunk and hand movements cause a degraded estimate of the global trunk heading.

\section{CONCLUSION / DiscuSSION}

This paper presents a method to accurately estimate the position and orientation of the hand with respect to the trunk and simultaneously estimate the global orientation of the trunk.

Change in position and orientation can be estimated using inertial sensors for short intervals. We aided the inertial sensors by attaching a permanent magnet to the hand and measuring the induced field using set of magnetometers attached to the trunk which allows us to estimate drift free positions and orientation in dynamic tasks over long periods.

The proposed tight filter approach (EKF) is able to compensate for both orientation and position drift. In addition, a good kinematic estimate is still obtained if magnetic information is temporarily unavailable as the filter will rely more on inertial sensing.
The results obtained are promising and can be compared to studies in which an actuated system was used to generate magnetic fields [5] [21]. However, it should be noted that the movement bandwidth was much lower in those studies (approximately 10 times) because the hardware did not allow to generate the magnetic pulses at an adequately high frequency $(>2 \mathrm{~Hz})$. In addition, under more complex movement conditions (varying trunk and hand) our system was able to estimate the positions more accurately whereas the relative orientations estimates were comparable. Finally, using a permanent magnet instead of actuating coils does not require actuation energy, which is an important advantage, bearing in mind that energy capacity is an important aspect when signals must be measured ambulatory.

The current setup allows accurate kinematic estimates for hand reaching tasks if the hand trunk distance is repeatedly (intervals less than 4 seconds) below $30 \mathrm{~cm}$. Hence, the accuracy of estimated position strongly depends on the SNR which is proportional to the distance of the permanent magnet with respect to the magnetometers. This is also demonstrated during the first movement condition in which the subject was asked to reach their arm maximally. The SNR dropped significantly during those maximum hand position phases $(50.1 \pm 10.0 \%)$ which subsequently resulted in a relatively low position accuracy $(19.6 \pm 4.6 \mathrm{~mm})$.

The accuracy of the relative hand trunk orientation strongly depends on the movement complexity. As indicated by the box plots, a non moving trunk (condition 1) results in the most accurate estimate of the relative hand trunk orientation. 
Similarly, for the global trunk orientation, an increased movement complexity results in a degraded orientation estimate. However, the first and second movement condition show a similar global trunk performance which can be explained by the fact that a substantial part of the error is contributed by the angle around the global vertical.

Increasing the measurement volume is possible by either adding extra magnetometers or applying a stronger magnet. However, it should be noted that the magnet dimensions become really big with respect to the size of the hand, when a distance over $70 \mathrm{~cm}$ is to be covered, because the field strength decreases cubically over distance, whereas the magnet's volume scales linearly.

Only one permanent magnet type was used in this study. Further research should be performed to find the most ideal magnet geometry for the application proposed. In addition, the dipole field approximation is optimal for specifically shaped magnets [34]. Therefore, if the magnet's geometry would be confined, tracking accuracy could be improved presumably.

Another solution to improve the SNR is using more sensitive magnetometers. The next generation magneto resistive (MR) sensors include Giant (GMR) and Tunnel (TMR) magneto resistive sensors which both have a higher sensitivity compared to the AMR based magnetometers that have been used in this study [35].

We used a constellation of four magnetometers, which were rigidly attached to the chest via a plexi plane. However, reducing the number of magnetometers needed would greatly improve the relevance of the proposed method in an ambulatory setting. Wahlstrom et al. [23] demonstrated that only a minimum of two 3D magnetometers is required to distinguish between changes in magnetic field induced by the permanent magnet or due to environmental field changes. That means the proposed configuration by Wahlstrom et al. obeys observability of the position and orientation states without using inertial sensors. Moreover, the potential of using a single trunk magnetometer aided by inertial sensing is demonstrated and shown in Fig. 5b and Fig. 7 However, reducing the number of magnetometers requires further research as the accuracy of estimated kinematic variables with a single magnetometer was low compared to a four magnetometer configuration.

If the magnetometers are directly attached to the body, for instance on the sternum, soft tissue artefacts could occur resulting in estimation errors. This could be mitigated when a single trunk magnetometer is used or when the filter is modified such that calibration parameters are estimated online.

Still, further research is required to see the effects of both spatial and temporal magnetic disturbances, soft tissue artefacts as well as the optimal strap location of magnetometers, especially if only one or two magnetometers are used.

Robustness could be further improved by adding biomechanical knowledge of the consecutive links. If the orientation of the shoulder, upper and lower arm is known, forward kinematics can be used to predict the position of the hand with respect to the trunk [12].

The trunk orientation is estimated using the common field component measured by each magnetometer and inclination information measured by the local accelerometer. If environmental magnetic disturbances affect only a part of the magnetometer grid an erroneous trunk heading and eventually relative pose is obtained. Hence, the filter could be improved such that those local disturbances are detected for each magnetometer and discarded if needed.

A final suggestion is to modify the filter such that various parameters can be estimated online. This includes the magnet dipole moment, magnet position with respect to the local inertial sensors and the relative magnetometer poses. A suggestion would be to use an Expectation Maximisation (EM) approach which is able to estimate parameters and states in parallel.

In conclusion, the proposed wearable measurement configuration of inertial and magnet sensors, combined with a permanent magnet on the hand is able to accurately estimate hand position and orientation with respect to the trunk and the global orientation of the trunk using inertial sensors, magnetometers and a permanent magnet. (a) Difference in hand-trunk distance.

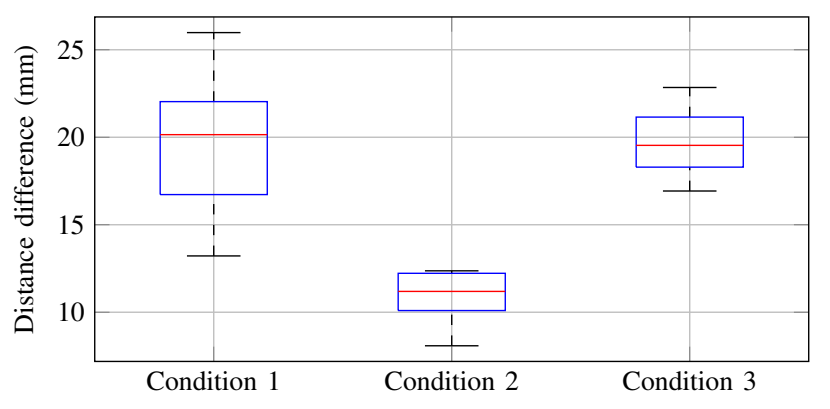

(b) Difference in hand-trunk (RO) and global (GO) orientation.

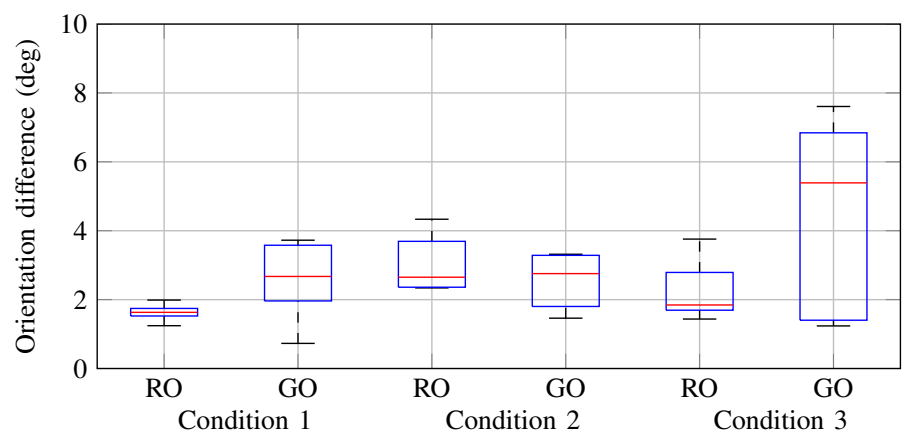

Fig. 8: Box whisker plots of the estimated kinematic variables. The columns refer to the three different measurement conditions. The box has lines at the lower quartile, median and higher quartile values. The whiskers are the lines showing the extend of the rest of the data. The difference in distance (left) and total orientation (right) are given. The difference in orientation is given for both global (GO) and relative orientation (RO). 


\section{APPENDIX}

A. Partial derivative of the magnetic measurement function w.r.t the position (superscripts are omitted for clarity):

$$
\begin{aligned}
\frac{\partial \boldsymbol{y}_{k, m a g}^{l}}{\partial \boldsymbol{r}} & =3 \frac{\partial}{\partial \boldsymbol{r}}\|\boldsymbol{r}\|_{2}^{-5}\left(\boldsymbol{r}^{T} \boldsymbol{m}\right) \boldsymbol{r}-\frac{\partial}{\partial \boldsymbol{r}}\|\boldsymbol{r}\|_{2}^{-3} \boldsymbol{m} \\
& =\frac{3}{\|\boldsymbol{r}\|_{2}^{5}}\left(\left(\boldsymbol{r}^{T} \boldsymbol{m}\right) I_{3}+\boldsymbol{r} \boldsymbol{m}^{T}-\frac{5\left(\boldsymbol{r}^{T} \boldsymbol{m}\right) \boldsymbol{r} \boldsymbol{r}^{T}}{\boldsymbol{r}^{T} \boldsymbol{r}}+\boldsymbol{m} \boldsymbol{r}^{T}\right)
\end{aligned}
$$

B. Partial derivatives of the magnetic measurement with respect to state:

$$
\begin{aligned}
\frac{\partial \boldsymbol{y}_{k, m a g}^{l}}{\partial \boldsymbol{p}}= & \frac{3}{\left\|\boldsymbol{r}^{l}\right\|_{2}^{5}}\left(\left(\boldsymbol{r}^{l, T} \boldsymbol{m}\right) I_{3}+\boldsymbol{r}^{l} \boldsymbol{m}^{T}\right) \\
& -\frac{3}{\left\|\boldsymbol{r}^{l}\right\|_{2}^{5}}\left(\frac{5\left(\boldsymbol{r}^{l, T} \boldsymbol{m}\right) \boldsymbol{r}^{l} \boldsymbol{r}^{l, T}}{\boldsymbol{r}^{l, T} \boldsymbol{r}^{l}}+\boldsymbol{m} \boldsymbol{r}^{l, T}\right) \\
\frac{\partial \boldsymbol{y}_{k, m a g}^{l}}{\partial \boldsymbol{v}}= & 0_{3} \\
\frac{\partial \boldsymbol{y}_{k, m a g}^{l}}{\partial \delta \boldsymbol{\theta}^{b t}}= & -J\left(\boldsymbol{r}_{l}^{b}\right) \bar{R}^{b t}\left[R^{t m} \boldsymbol{m}^{m}\right]_{\times} \\
& -R^{b t}\left(I_{3}+[\theta]_{\times}^{b t}\right) R^{t m} \boldsymbol{m}^{m}\left(J\left(\boldsymbol{r}_{l}^{b}\right) R^{b t} \boldsymbol{p}_{m}^{b}\right)^{T} \\
\frac{\partial \boldsymbol{y}_{k, m a g}^{l}}{\partial \boldsymbol{B}^{g}}= & R^{g s, T} \\
\frac{\partial \boldsymbol{y}_{k, m a g}^{l}}{\partial \boldsymbol{b}_{a}^{t}}= & 0_{3} \\
\frac{\partial \boldsymbol{y}_{k, m a g}^{l}}{\partial \delta \boldsymbol{b}_{g}^{t}}= & 0_{3} \\
\frac{\partial \boldsymbol{y}_{k, m a g}^{l}}{\partial \delta \boldsymbol{\theta}^{g s}}= & \bar{R}^{g s, T}\left[\boldsymbol{B}^{g}\right]_{\times} \\
\frac{\partial \boldsymbol{y}_{k, m a g}^{l}}{\partial \delta \boldsymbol{b}_{g}^{b}}= & 0_{3} .
\end{aligned}
$$

\section{Expression of the error angle propagation}

One can find the following expression for the error angle dynamics [27] [36]:

$$
\dot{\delta \theta}=-[\hat{\omega}]_{\times} \delta \theta+\delta \omega
$$

where $\delta \boldsymbol{\theta}$ is the error angle and $\hat{\omega}$ is the estimated angular velocity, and $\delta \omega$ is defined as:

$$
\begin{aligned}
\delta \boldsymbol{\omega} & =\boldsymbol{\omega}-\hat{\boldsymbol{\omega}} \\
& =\left(\boldsymbol{y}_{g y r}-\boldsymbol{b}-\boldsymbol{e}\right)-\left(\boldsymbol{y}_{g y r}-\hat{\boldsymbol{b}}\right) \\
& =-\delta b-e .
\end{aligned}
$$

Discretizing (33) using a zero order hold assumption with sample period $T$ and neglecting the noise term $e$, gives:

$$
\delta \boldsymbol{\theta}_{k+1}=\left(I_{3}-\left[T \hat{\boldsymbol{\omega}}_{k}\right]_{\times}\right) \boldsymbol{\delta} \boldsymbol{\theta}_{k}-T \delta \boldsymbol{b} .
$$

\section{REFERENCES}

[1] H. Zhou and H. Hu, "Human motion tracking for rehabilitation-A survey," Biomedical Signal Processing and Control, vol. 3, no. 1, pp. $1-18,2008$.

[2] G. Welch and E. Foxlin, "Motion tracking survey," IEEE Computer Graphics and Applications, 2002.

[3] H. Luinge, P. Veltink, and C. T. M. Baten, "Ambulatory measurement of arm orientation." Journal of Biomechanics, vol. 40, no. 1, pp. 78-85, 2007.

[4] X. Yun and E. Bachmann, "Design, implementation, and experimental results of a quaternion-based Kalman filter for human body motion tracking," Robotics, IEEE Transactions on, vol. 22, no. 6, pp. 12161227, 2006.

[5] D. Roetenberg, P. Slycke, and P. Veltink, "Ambulatory position and orientation tracking fusing magnetic and inertial sensing," IEEE Transactions on Biomedical Engineering, vol. 54, no. 5, pp. 883-890, Jan. 2007.

[6] R. Zhu and Z. Zhou, "A real-time articulated human motion tracking using tri-axis inertial/magnetic sensors package," Neural Systems and Rehabilitation Engineering, IEEE Transactions on, vol. 12, no. 2, pp. 295-302, 2004.

[7] A. M. Sabatini, "Estimating Three-Dimensional Orientation of Human Body Parts by Inertial/Magnetic Sensing," Sensors, vol. 11, no. 2, pp. 1489-1525, Feb. 2011.

[8] P. Veltink, F. v. Meulen, B. v. Beijnum, B. Klaassen, H. Hermens, E. Droog, M. Weusthof, F. Lorussi, A. Tognetti, J. Reenalda, C. Nikamp, C. Baten, J. Buurke, J. Held, A. Luft, H. Luinge, G. De Toma, C. Mancusso, and R. Paradiso, "Daily-life tele-monitoring of motor performance in stroke survivors," accepted conferernce article 3DAHM Laussanne 2014.

[9] I. Skog, P. Händel, J. Nilsson, and J. Rantakokko, "Zero-velocity detection - an algorithm evaluation." IEEE transactions on bio-medical engineering, vol. 57, no. 11, pp. 2657-2666, Nov. 2010.

[10] H. M. Schepers, H. F. J. M. Koopman, and P. H. Veltink, "Ambulatory assessment of ankle and foot dynamics," IEEE Transactions on Biomedical Engineering, vol. 54, no. 5, pp. 895-902, May 2007.

[11] P. Veltink, H. Kortier, and H. Schepers, "Sensing Power Transfer Between the Human Body and the Environment," Biomedical Engineering, IEEE Transactions on, vol. 56, no. 6, pp. 1711-1718, 2009.

[12] D. Roetenberg, H. Luinge, and P. Slycke, "Xsens MVN: full 6DOF human motion tracking using miniature inertial sensors," Xsens Motion Technologies BV, Tech. Rep, 2009.

[13] H. Zhou and H. Hu, "Reducing drifts in the inertial measurements of wrist and elbow positions," Instrumentation and Measurement, IEEE Transactions on, vol. 59, no. 3, pp. 575-585, 2010.

[14] D. Roetenberg and P. Veltink, "Camera-marker and inertial sensor fusion for improved motion tracking," Gait \& posture, vol. 22, pp. 1-53, 2005.

[15] E. Foxlin, M. Harrington, and G. Pfeifer, "Constellation: A wide-range wireless motion-tracking system for augmented reality and virtual set applications," ... of the 25th annual conference on ..., 1998.

[16] E. Foxlin, "Pedestrian tracking with shoe-mounted inertial sensors," Computer Graphics and Applications, 2005.

[17] J. Hol, F. Dijkstra, H. Luinge, and T. B. Schon, "Tightly coupled UWB/IMU pose estimation," in Ultra-Wideband, 2009. ICUWB 2009. IEEE International Conference on. IEEE, 2009, pp. 688-692.

[18] J. Hol, T. B. Schon, H. Luinge, P. J. Slycke, and F. Gustafsson, "Robust real-time tracking by fusing measurements from inertial and vision sensors," Journal of Real-Time Image Processing, vol. 2, no. 2-3, pp. 149-160, 2007.

[19] F. H. Raab, E. B. Blood, T. O. Steiner, and H. R. Jones, "Magnetic Position and Orientation Tracking System," Aerospace and Electronic Systems, IEEE Transactions on, no. 5, pp. 709-718, 1979.

[20] H. Schepers and P. Veltink, "Stochastic magnetic measurement model for relative position and orientation estimation," Measurement Science and Technology, vol. 21, p. 065801, 2010.

[21] H. Schepers, D. Roetenberg, and P. Veltink, "Ambulatory human motion tracking by fusion of inertial and magnetic sensing with adaptive actuation," Medical and Biological Engineering and Computing, vol. 48 , no. 1, pp. 27-37, 2010.

[22] M. Birsan, "Unscented particle filter for tracking a magnetic dipole target," in Proceedings of MTS/IEEE OCEANS, 2005, 2005.

[23] N. Wahlstrom, J. Callmer, and F. Gustafsson, "Magnetometers for tracking metallic targets," in Information Fusion (FUSION), 2010 13th Conference on, 2010, pp. 1-8. 
[24] F. Gustafsson and N. Wahlstrom, "Method and device for pose tracking using vector magnetometers," Dec. 5 2013, wO Patent App. PCT/IB2013/001,229. [Online]. Available: https://www.google. com/patents/WO2013150385A3?cl=en

[25] A. Vaccarella, E. de Momi, A. Enquobahrie, and G. Ferrigno, "Unscented Kalman filter based sensor fusion for robust optical and electromagnetic tracking in surgical navigation," IEEE Transactions on Instrumentation and Measurement, vol. 62, no. 7, pp. 2067-2081, 2013.

[26] F. Gustafsson, Statistical sensor fusion. Lund: Studentlitteratur, 2012.

[27] J. Crassidis, F. Markley, and Y. Cheng, "Survey of nonlinear attitude estimation methods," Journal of Guidance Control and Dynamics, vol. 30, no. 1, p. 12, 2007.

[28] J. Kuipers, Quaternions and rotation sequences. Princeton university press Princeton, NJ, USA:, 1999.

[29] J. Antonsson, "Motion tracking using a permanent magnet," Master's thesis, Linkoping, 2013

[30] [Online]. Available: http://www.xsens.com/products/ mtw-development-kit/

[31] M. Kok, J. Hol, T. B. Schon, F. Gustafsson, and H. Luinge, "Calibration of a magnetometer in combination with inertial sensors," in 15th International Conference on Information Fusion, FUSION 2012, 2012, pp. 787-793.

[32] S. Bonnet, C. Bassompierre, C. Godin, S. Lesecq, and A. Barraud, "Calibration methods for inertial and magnetic sensors," Sensors and Actuators A: Physical, vol. 156, no. 2, pp. 302-311, 2009.

[33] K. Parsa, J. Angeles, and A. K. Misra, "Rigid-body pose and twist estimation using an accelerometer array," Archive of Applied Mechanics, vol. 74, no. 3-4, pp. 223-236, 2004.

[34] A. J. Petruska and J. J. Abbott, "Optimal permanent-magnet geometries for dipole field approximation," IEEE Transactions on Magnetics, vol. 49, no. 2, pp. 811-819, 2013.

[35] A. Jander, C. Smith, and R. Schneider, "Magnetoresistive sensors for nondestructive evaluation (Invited Paper)," Nondestructive Evaulation for Health Monitoring and Diagnostics, vol. 5770, pp. 1-13, 2005.

[36] H. G. Kortier, V. I. Sluiter, D. Roetenberg, and P. H. Veltink, "Assessment of hand kinematics using inertial and magnetic sensors." Journal of NeuroEngineering and Rehabilitation, vol. 11, no. 1, p. 70, Apr. 2014.

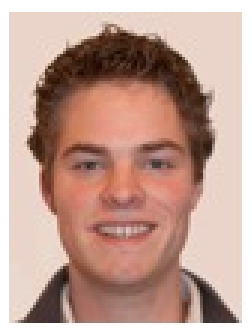

Henk G. Kortier received the MSc degree in Electrical Engineering from the University of Twente, Enschede, the Netherlands. He has been working as a $\mathrm{PhD}$ student at the biomedical signals and system group at the University of Twente since 2010. This PhD work is part of the STW 'PowerSensor' project which aims for the development of a glove instrumented with miniature inertial, magnetic and force/torque sensors. Eventually, the glove will be used to assess the hand interaction in ADL tasks.

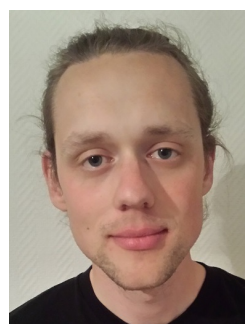

Jacob Antonsson received the MSc degree in engineering biology from Linkping University, Sweden. Since 2013 he has been working as a PhD student at the automatic control department at Lund University, Sweden. His research interests include inference in non-linear state space models, inference in high-dimensional models and machine learning and sensor fusion in general.

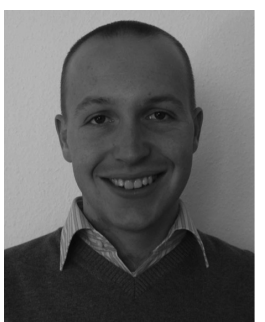

H. Martin. Schepers received the MSc degree in 2004 and the $\mathrm{PhD}$ degree in 2009, both in Electrical Engineering at the University of Twente, Enschede, the Netherlands. His $\mathrm{PhD}$ work was part of the FreeMotion project, which researches accurate and reliable methods of ambulatory motion analysis to provide practical decision making tools to a large group of professionals in health care, ergonomics and sports. After his $\mathrm{PhD}$, he started working within the Research department at Xsens Technologies B.V., where he is currently working as a Lead Algorithms Engineer on motion capture technology based on fusion of inertial sensing with various aiding sensor technologies.

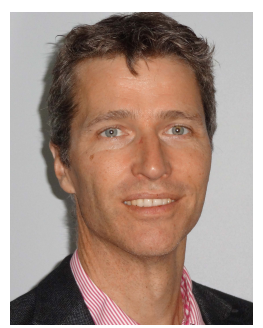

Fredrik Gustafsson is professor in sensor informatics at the Linköping University. His research interests are in stochastic signal processing, adaptive filtering, and change detection, with applications to communication, vehicular, airborne, and audio systems. His work in the sensor fusion area involves design and implementation of nonlinear filtering algorithms for localization, navigation, and tracking of all kind of platforms. He is a cofounder of the companies NIRA Dynamics and Softube, developing signal processing software solutions for automotive and music industry, respectively. Dr. Gustafsson was an Associate Editor for the IEEE Transactions Of Signal Processing from 2000 to 2006 and is currently Associate Editor for the EURASIP Journal on Applied Signal Processing and International Journal of Navigation and Observation.

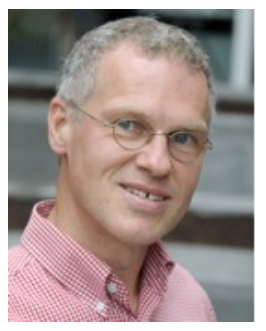

Peter H. Veltink is professor of technology for the restoration of human function at the University of Twente. His research interests are in Biomechatronics and Neural Engineering. His research includes ambulatory sensing of human movements, artificial human motor control and neurostimulation. Prof. Veltink has been an Associate Editor for the IEEE Transactions of Neural Systems and Rehabilitation Engineering until 2014. He is the (co)author of over 125 peer reviewed journal papers. Prof. Veltink has been the scientific coordinator of three EU research training networks and currently coordinates the EU project INTERACTION since November 2011. 